\title{
From SPS to RHIC: Maurice and the CERN heavy-ion programme
}

\author{
Ulrich Heinz \\ Physics Department, The Ohio State University, Columbus, Ohio 43210, USA 1 \\ and \\ CERN, Physics Department, Theoretical Physics Division, \\ CH-1211 Geneva 23, Switzerland
}

\begin{abstract}
Maurice Jacob played a key role in bringing together different groups from the experimental and theoretical nuclear and particle physics communities to initiate an ultrarelativistic heavy-ion collision program at the CERN SPS, in order to search for the quark-gluon plasma. I review the history of this program from its beginnings to the time when the Relativistic Heavy Ion Collider (RHIC) at Brookhaven National Laboratory (BNL) started operation. I close by providing a glimpse of the important discoveries made at RHIC and giving an outlook towards heavy-ion collisions at the Large Hadron Collider (LHC). During Maurice's life and not least through his perpetually strong influence, relativistic heavy-ion physics has matured and led to discoveries that radiate into many other fields of physics. Heavy-ion physicists owe a great deal to Maurice Jacob.
\end{abstract}

Invited talk presented at the "Maurice Jacob Memorial Meeting", CERN, 11 September 2007

\footnotetext{
${ }^{1}$ Permanent address. Email: heinz@mps.ohio-state.edu. Work supported by the U.S. Department of Energy under contract DE-FG02-01ER41190.
} 


\section{Introduction}

I have been asked to talk at this "Maurice Jacob Memorial Meeting" about Maurice's role in relativistic heavy-ion physics. I am a little younger than the other speakers, and my first serious contact with Maurice didn't happen until early 1987 when, as Leader of the CERN Theory Division, he offered me a junior staff position at CERN. This was just the time when the CERN heavy-ion programme had finished its first run, closely followed by a similar program at the AGS at BNL (where I was then working), while BNL scientists were already busy trying to obtain approval to build a dedicated heavy-ion collision facility, the Relativistic Heavy Ion Collider, at 10 times the energy of the CERN SPS. Little did I know that Maurice was far ahead of them and already thinking about heavy ions in the LHC, at 30 times the energy of RHIC! Neither did I accept CERN's offer, nor did I stay at BNL - instead, I joined the faculty at the University of Regensburg where I started to build my own heavy-ion theory group. But Maurice made sure that I spent many springs and summers at CERN, as a regular visitor and a member of the SPS program committee, and during these times we, together with Leon van Hove, spent endless hours discussing the new heavy-ion data and their possible interpretations. It was a heady time of excitement, confusion and discovery as we were groping our way through the complexities of the theoretical problems posed by the extraordinarily tiny, short-lived, extremely dense and highly dynamical "fireballs" created in these nuclear collisions. In 1998 I went on a 3-year leave of absence from Regensburg to join the CERN TH staff and coordinate their heavy-ion theoretical activities just when the second phase of the SPS heavy-ion programme, the lead beam programme, burst into full bloom. As it turned out, I never returned to Regensburg, but instead followed a call from the US in late 2000 when I joined the faculty of The Ohio State University just after RHIC had finally been completed and finished its first run.

When I came to CERN, Maurice had already officially retired (which simply meant he was travelling more in his various international leadership roles on which others report elsewhere in this volume). But whenever he was home, he looked me up to "talk physics". As the completion of RHIC drew closer, CERN heavyion physicists got together to try to assess the results of the CERN heavy-ion programme as it was winding down and before the limelight would move across the atlantic. As the discussions heated up (see below), it was entirely natural that Maurice was "called back" from retirement and asked to write (together with me) a "White Paper" summarizing the achievements and insights made at the SPS. A special seminar and press conference followed on Feb. 10, 2000, where

CERN announced ... - but I am getting ahead of myself! Let us start at the beginning ... 


\section{The SPS heavy-ion programme 1986-2003}

Heavy ion collisions at relativistic energies $\left(E_{\text {beam }}>A m_{N}\right)$ were first explored in the mid 1970s at the BEVALAC at Berkeley, motivated by curiosity about the properties of nuclear matter at densities much above that of atomic nuclei and by theoretical speculations about abnormal states of matter at several times nuclear equilibrium density [1]. As QCD, the modern theory of strong interactions, became more widely known, it was discovered that it predicted a phase transition from hadronic matter to a plasma of color-deconfined quarks and gluons, the quark-gluon plasma, at high densities and temperatures [2]. But the beams provided by the BEVALAC were not energetic enough to reach this new state of matter. How to get higher energy heavy ion beams without breaking the bank? Scientists started looking at the possibility of injecting heavy ions into existing higher energy proton accelerators, such as the AGS at Brookhaven and the CERN SPS. Maurice played a decisive role at CERN in bringing together different groups, experimentalists and theorists, from the nuclear and particle physics communities to initiate an ultra-relativistic $\left(E_{\text {beam }} \gg A m_{N}\right)$ heavy-ion collision program at CERN, and to secure the support of the laboratory management for this adventure at a time when CERN was building LEP. The key move was the proposal to build the heavy-ion program using existing equipment not only for the accelerator, but also for the ion source and detectors. In 1986 the SPS delivered for the first time ${ }^{16} \mathrm{O}$ beams of $60 \mathrm{~A} \mathrm{GeV}$ to a handful of experiments, followed in subsequent years by ${ }^{32} \mathrm{~S}$ beams at 60 and $200 \mathrm{AGeV}$ which were used to study $\mathrm{S}+\mathrm{S}, \mathrm{S}+\mathrm{U}$ and $\mathrm{S}+$ emulsion collisions, as well as a few other target nuclei. This phase lasted from 1986 to 1993.

The collisions produced unprecedented large numbers of secondary particles whose energy spectra showed tantalizing evidence for thermal multi-particle production at high temperatures, with an enhanced probability for creating strange hadrons. But since the experiments were using second hand equipment they were not optimized for the task. It proved difficult to test theoretical predictions with experiments where different observables were measured in different regions of phase space, and the limited experimental acceptance required large acceptance corrections which made it tedious to compare data between different experiments where there acceptances overlapped. Every exciting observation (and, as expected when you explore uncharted territory, there were many!) thus raised more questions than it answered. Furthermore, sulfur nuclei were kind of small "heavy" ions.

Scientists thus started building a new ion source for lead beams and a second generation of large, dedicated multi-purpose heavy-ion detectors, which went into operation in 1994 with a $160 A \mathrm{GeV}$ beam of ${ }^{207} \mathrm{~Pb}$. The CERN Pb-beam program lasted almost 10 years, finishing in 2002 with a low energy run at 40, 30 and 20 $\mathrm{GeV} /$ nucleon. When the SPS was running protons instead of heavy-ions, the experiments collected $p p$ and $p A$ reference data. A final heavy-ion run with an 
indium beam, which the NA60 experiment used to study ${ }^{115} \mathrm{In}+\mathrm{In}$ collisions at $160 \mathrm{~A} \mathrm{GeV}$, yielded an outstanding set of data on electromagnetic signatures from the collision fireball that are even now keeping a spotlight on CERN while much of the attention of the heavy-ion community has moved to RHIC.

I looked up on the CERN web site the list of heavy-ion experiments completed during this 15-year period. I counted 25 large-detector experiments (including 7 big multi-year efforts) plus another 20 or so emulsion experiments. (A parallel effort at the BNL AGS with beam energies ranging from 2 to $15 \mathrm{GeV} /$ nucleon, providing ${ }^{28} \mathrm{Si}$ beams from 1987 and ${ }^{197} \mathrm{Au}$ beams from 1995 to 1999, was the second leg of the relativistic heavy ion program during the final decades of the last century.) Clearly, the CERN heavy-ion program (which without Maurice might never have happened) ended up being a very strong and successful operation which broadened CERN's research portfolio and strengthened its reputation as the leading accelerator facility in the world. And without the experience gained in the CERN heavy-ion program, RHIC could never have become the immediate smashing success that it did.

The incoming SPS data were new and exciting. We saw more than a 1000 produced particles per central collision event. Each event was its own statistical ensemble, exhibiting collective dynamics - thermodynamics and hydrodynamics became the standard language for talking about the collision dynamics. Compared to proton-proton collisions, strange particle production was enhanced while $J / \psi$ mesons and other charmonium states were suppressed. The final hadron spectra were thermal and showed strong, anisotropic collective flow, reflecting expansion transverse to the beam direction with more than half the speed of light. It was clear that we had succeeded in creating bulk matter made of strongly-interacting constituents, and had turned into a new breed of condensed matter physicists studying the collective properties and phase diagram of such matter!

But, as nice as the data were, their interpretation was difficult: One was dealing with a very complex, highly dynamical system of which each detector measured only a (in some cases small) subset of observables. Where measurements overlapped, the data did not immediately agree with each other. Information how the various interesting observations correlated with each other was initially quite sketchy. As a result, first opinions varied widely, and convergence was slow; in hindsight I can say that all the right ideas were there, but parsing incorrect concepts and interpretations took time.

\section{Maurice, the beacon}

During this time, Maurice's main responsibilities lay elsewhere. As recounted by others at this meeting, he was busy directing the CERN Theory Division, planning for LEP and the LHC, editing journals, books and proceedings, and promoting research worldwide in various leading roles in the French and Euro- 
pean Physical Societies, the European Space Agency, etc. Still, as a responsible and supportive father, he followed the growth of his child, the CERN heavy-ion programme, carefully. In continuous discussions with colleagues, and as an active conference participant and highly-sought speaker, he tried to distill his own picture of the heavy-ion fireball evolution from the data. In numerous invited opening and summary talks he captured the excitement of the field, encouraged the young generation of scientists entering the field in droves, and stimulated his colleagues with his insights, speculations and proposals.

Typically, when the field approached a critical transition point, he was "called upon" for his assessment and advice. Here is an example from an opening talk that he gave at the 5th Conference on Nucleus-Nucleus Collisions in Taormina in June 1994, when CERN was making the transition from $\mathrm{S}$ to $\mathrm{Pb}$ beams and BNL switched from Si to Au beams:

"There is no doubt that a new state of matter, with a density of at least an order of magnitude higher than hadronic matter, is created in heavy ion collisions. We do not yet know what it is and whether or not there is a phase transition between two very different forms of dense matter, as it is expected from Quantum Chromodynamics. Nevertheless, since we are after a phase transition there is nothing like volume and time and also, in order to increase time, collision energy. Since we have good proven tools now at hand, we can expect much from an increase in volume and an increase in collision energy. We can therefore approach with enthusiasm the new rounds of experiments."

Clearly, Maurice was seeing farther than many of his contemporaries: not only did he have "no doubt" where others were still entrenched in hot debates, but he was already setting the stage not only for the CERN lead beam programme, but also for higher energies at RHIC and LHC!

\section{Preparing the CERN press release}

In 1998, Maurice retired from CERN. The completion of RHIC at BNL was looming, with first $\mathrm{Au}+\mathrm{Au}$ collision at $200 \mathrm{GeV} /$ nucleon in the center of mass (!) planned for 1999. (A series of major leaks in the old cryogenic system inherited from the cancelled ISABELLE project delayed RHIC start-up by another year.) A number of scientists at CERN, in particular Reinhard Stock from the University of Frankfurt, pushed for a public statement by CERN that would summarize the achievements and assess the success of the CERN heavy-ion program before RHIC went into operation. What could (and should) be said at the eve of the RHIC era? No doubt the CERN SPS program, and the pioneers who had driven it, against an adverse tide of scientific and political difficulties, had made tremendous advances towards the ultimate goal - creating quark-gluon plasma in the laboratory. This deserved recognition. But many detailed questions were still 
discussed controversially, without hope for final resolution at the SPS because its beam energy was limited (see below). Ignoring this and claiming unqualified discovery of the QGP with the incomplete evidence in hand would do irreparable damage to the field (not to speak of the wrath of the colleagues at RHIC!). All were acutely aware of the political and sociological aspects of this assessment.

I joined the CERN Theory Division in August 1998 and was immediately drafted to help coordinate the assessment process. In September of that year leading participants of the program met for a retreat in Chamonix. We had about 4-5 months worth of lead beam data to look at for our assessment, with more on tape but not yet analyzed. (Typically, the heavy-ion runs lasted for 4-6 weeks every year.) The discussions at the retreat were contentious and revealed a need for consolidation. We went to work. In March 1999 I was asked to give an SPS heavy-ion status report to the SPSC; clearly, the lab management had made it a priority to take stock of the return on their investment.

In late summer 1999, another "call back to duty" was issued to Maurice: he was asked to coordinate, together with me, the writing of a "White Paper" summarizing the scientific achievements of the CERN Pb-beam program that reflected the consensus of the community. It was titled "Evidence for a new state of matter: An assessment of the results from the CERN lead beam programme" [4]. I have heard the process leading to this document described sarcastically as a "discovery by committee"; the truth is that a group of intellectual leaders, in perfect scientific tradition, got together and sorted the empirical and theoretical evidence to extract a coherent and compelling overall picture, throwing out claims and speculations that, based on the available facts, didn't hold water. Maurice Jacob was the perfect mediator for this process. The resulting White Paper reflected the consensus of the SPS heavy-ion community to the extent that consensus could be reached; what was not consensus was not written down.

You could ask: Why was this so difficult? The answer is given in [4]:

"We emphasize that the evidence collected so far is "indirect" since it stems from the measurement of particles which have undergone significant reinteractions between the early collision stages and their final observation. Still, they retain enough memory of the initial quark-gluon state to provide evidence for its formation, like the grin of the Cheshire Cat in Alice in Wonderland which remains even after the cat has disappeared. It is expected that the present "proof by circumstantial evidence" for the existence of a quark-gluon plasma in high energy heavy ion collisions will be further substantiated by more direct measurements (e.g. electromagnetic signals which are emitted directly from the quarks in the QGP) which will become possible at the much higher collision energies and fireball temperatures provided by RHIC at Brookhaven and later the LHC at CERN."

I should add another complication: As we now know and is shown in Fig. 1, the SPS beam energy was just barely enough to reach the quark-gluon plasma phase: 


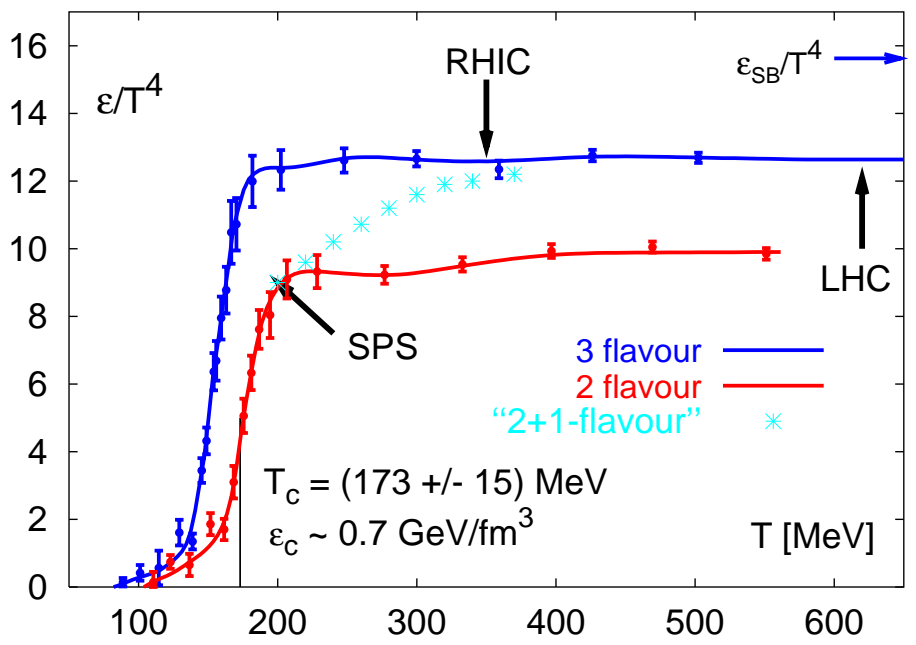

Figure 1: Normalized energy density $\varepsilon / T^{4}$ vs. temperature $T$ from Lattice QCD [5], with arrows indicating the temperatures and energy densities reached at SPS, RHIC and (presumably) LHC. The steep rise at $T_{c}$ indicates the phase transition from a hadron gas to the quark-gluon plasma.

the SPS experiments were "living on the edge". In consequence, the QGP phase lasted only for a very short time, converting to hadrons almost immediately, and most of what was observed were manifestations of collective hadron dynamics. They camouflaged whatever QGP signatures there were, making it difficult to extract the latter in an unambiguous fashion.

In the end we wrote "It walks like a duck, it quacks like a duck, ..." but didn't complete the sentence [4]:

"A common assessment of the collected data leads us to conclude that we now have compelling evidence that a new state of matter has indeed been created, at energy densities which had never been reached over appreciable volumes in laboratory experiments before and which exceed by more than a factor 20 that of normal nuclear matter. The new state of matter found in heavy ion collisions at the SPS features many of the characteristics of the theoretically predicted quark-gluon plasma."

Maurice and I received a lot of sharp-tongued comments about this carefully chosen "politically correct" formulation. We took it with humour. What is more important than a never-ending discussion of whether the new state of matter for which CERN claimed discovery was the long-sought QGP is that, in the process of analyzing the SPS data, we had obtained a pretty good picture of the timeevolution of the hot fireball [4]:

"In spite of its many facets the resulting picture is simple: the two colliding nuclei deposit energy into the reaction zone which materializes in the form of quarks 
and gluons which strongly interact with each other. This early, very dense state (energy density about $3-4 \mathrm{GeV} / \mathrm{fm}^{3}$, mean particle momenta corresponding to $T \approx 240 \mathrm{MeV}$ ) suppresses the formation of charmonia, enhances strangeness and begins to drive the expansion of the fireball. Subsequently, the "plasma" cools down and becomes more dilute. At an energy density of $1 \mathrm{GeV} / \mathrm{fm}^{3}(T \approx$ $170 \mathrm{MeV}$ ) the quarks and gluons hadronize and the final hadron abundances are fixed. At an energy density of order $50 \mathrm{MeV} / \mathrm{fm}^{3}(T=100-120 \mathrm{MeV})$ the hadrons stop interacting, and the fireball freezes out. At this point it expands with more than half the light velocity."

On 10 February, 2000, CERN held a special seminar [6], with talks from the major SPS heavy-ion experiments, were the discovery of "A New State of Matter created at CERN" was announced. I still have a copy of a volume put together by the CERN press office that contains press clippings from around the world in reaction to this announcement; it is several hundred pages thick.

\section{From SPS to RHIC}

Even with 20/20 hindsight, there is surprisingly little written in the SPS White Paper [4] that we would like to take back. Some specific phrases connected with the discussion of strangeness enhancement and $J / \psi$ suppression were exaggerated - data and theory hadn't quite settled yet when the paper was written. Overall, the first RHIC data that came in later in the summer of 2000 provided a spectacular confirmation of the picture that had been developed at the SPS. Of course, RHIC data went much beyond the observations at the SPS and, as I briefly discuss below, led to quite unexpected and exciting discoveries in their own right. But it is worth emphasizing that, in doing so, they didn't conflict with what had been found at the AGS and SPS, but built naturally upon the strong foundations laid at lower energies.

An interesting discussion is connected with Maurice's use of the phrase "quarks and gluons roaming freely" [4]:

"It has been expected that in high energy collisions between heavy nuclei sufficiently high energy densities could be reached such that this new state of matter would be formed. Quarks and gluons would then freely roam within the volume of the fireball created by the collision. ..."

It is a powerful phrase that, like the "grin of the Ceshire Cat" from the first quote above, was picked up by journalists around the world in their reports on the CERN press release. We meant it to mean "unshackled, freed from their hadronic prison of $\sim 1 \mathrm{fm}^{3}$ living space", but scientists (especially ones working at RHIC) frequently (mis)quote it with the meaning "moving almost without interactions, as in a dilute gas". It cannot be denied that the limitations of QCD 
perturbation theory for the description of the transport and flow properties of the QGP were not yet fully appreciated at the end of the SPS era and, as I will tell, RHIC brought about a real paradigm shift. But we definitely knew already before 2000 that the observed strong collective flow of the SPS "Little Bangs" required significant interactions among the fireball constituents. The White Paper [4] therefore makes specific reference to "quarks and gluons which strongly interact with each other" (see above). But, when misinterpreted in this particular way, "roaming freely" makes for a perfect backdrop to highlight the most spectacular discovery made at RHIC, namely that of the "perfect QGP liquid".

Before discussing this important RHIC result (that Maurice enjoyed very much) let me make a few sociological comments about the AGS-SPS-RHIC-LHC family. RHIC turned on in June 2000. The RHIC experiments produced results at a spectacularly fast rate. 20 years of struggle and consolidation at the SPS and AGS had led to the development of very successful and efficient concepts for the RHIC experiments and data analysis chains. Many of the key ideas developed at the SPS were confirmed at RHIC, enabling the RHIC scientists to quickly move further and facilitating the recognition of important new discoveries. Experience gained during the SPS program now informs the planning for the LHC heavy-ion experiments (ALICE, CMS, ATLAS), and new technologies developed for RHIC are flowing back across the Atlantic into the construction of LHC detectors. We thus hope for a similarly smooth and successful start of the LHC heavy-ion program next year, and I am sure Maurice would have loved to see the birth if this great-grand-child of his (especially for all the spectacular jets that it will throw about!).

Now let me return to the "perfectly liquid QGP" at RHIC. This is an interesting story whose long version can be read in various review articles (e.g. [9]). One chapter of this story is the observation of "jet quenching", i.e. the suppression of high- $p_{T}$ jets by the dense medium created in the collision fireball. This is, of course, something that excited Maurice tremendously, given his affinity to QCD jets throughout his life. I will here focus on a different part of the story, related to "elliptic flow". "Elliptic flow" denotes the anisotropic collective motion of particles produced in non-central heavy-ion collisions. If one pictures the two colliding nuclei as two spheres that smash into each other off-center, the part that actually interacts and gets stopped in the center-of-momentum frame is spatially deformed like an egg or almond whose long axis is oriented perpendicular to the reaction plane. After thermalization of the fireball matter inside that almond, the pressure gradient is larger in the short direction than in the long direction of the almond, so the matter is ejected with larger acceleration in the short direction. As a result, the final particles carry on average more momentum if they are parallel to the reaction plane than perpendicular to it. One measures this by Fourier analysing the angular distribution around the beam direction of the finally observed hadrons and extracting the second Fourier coefficient $v_{2}$. Figure 2 shows this so-called "elliptic flow coefficient" for a variety 
of different hadron species, as a function of their transverse momentum $p_{T}$ or transverse kinetic energy $\mathrm{KE}_{T}=m_{T}-m_{0}=\sqrt{p_{T}^{2}+m_{0}^{2}}-m_{0}$.
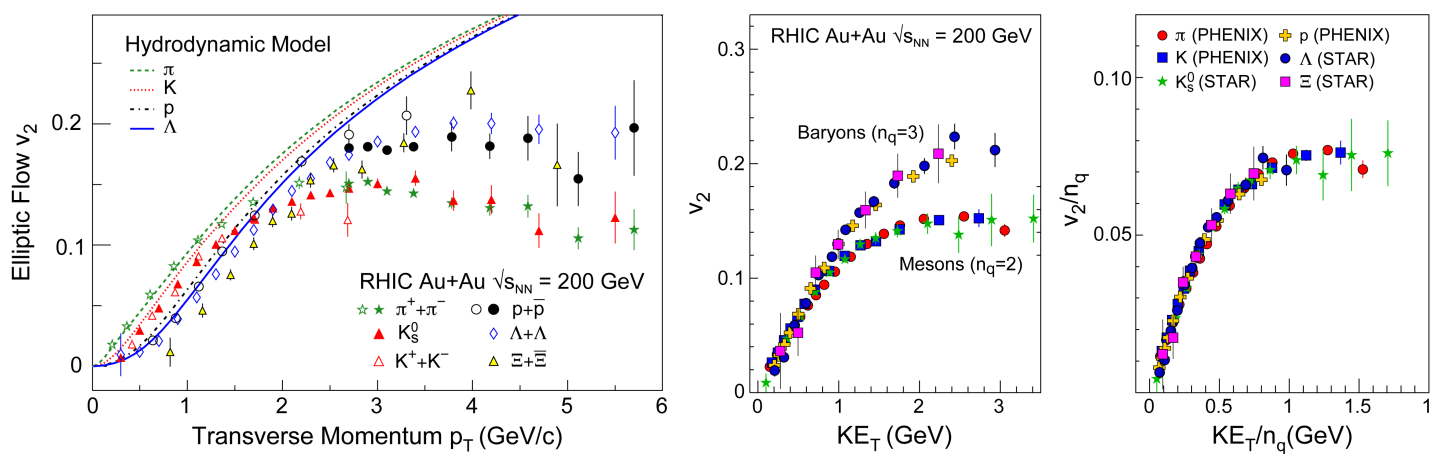

Figure 2: Left: Up to $p_{T} \sim 1.5 \mathrm{GeV} / c$, the differential elliptic flow $v_{2}\left(p_{T}\right)$ follows the hydrodynamical predictions for an ideal fluid perfectly [7]. Note that $>99 \%$ of all final hadrons have $p_{T}<1.5 \mathrm{GeV} / c$. Middle: When plotted against transverse kinetic energy, the differential elliptic flow follows different universal curves for mesons and baryons. Right: When scaled by the number of valence quarks, the differential elliptic flow per quark follows the same universal curve for all hadrons and for all values of (scaled) transverse kinetic energy $[8]$.

Observation of elliptic flow is exciting because it unambiguously demonstrates final state interactions among the produced particles: without interactions, there is no possibility to transform the initial geometric deformation in coordinate space into a final momentum-space anisotropy. In fact, for a given spatial deformation, the largest momentum-space anisotropy is expected for the most strongly interacting medium, i.e. for the shortest mean free path of the produced particles. In the limit of (approximately) zero mean free path, one expects thermalization to occur most rapidly, resulting in almost perfect hydrodynamic collective flow of the medium. Larger mean free paths would cause the fluid to be more viscous and the resulting momentum anisotropy to be smaller [10, 11]. The largest possible final $v_{2}$ is thus predicted by ideal fluid dynamics.

At the SPS, elliptic flow was measured in $\mathrm{Pb}+\mathrm{Pb}$ collisions and found to be about half of what ideal fluid dynamics predicts [12. But, as the left panel in Figure 2 shows, at RHIC the experimental data exhaust the ideal fluid prediction! Deviations from the hydrodynamic prediction occur only for the small fraction $(<1 \%)$ of hadrons whose transverse momenta are larger than $p_{T}>1.5 \mathrm{GeV}$. Furthermore, the data perfectly reproduce the hydrodynamically predicted dependence of $v_{2}\left(p_{T}\right)$ on the hadron rest masses. This thermal mass splitting can be (approximately) absorbed by plotting $v_{2}$ as a function of transverse kinetic energy instead of $p_{T}$ (middle panel in Fig. 2). Now all curves collapse, not only in the low- $p_{T}$ region where hydrodynamics works, but even at higher transverse kinetic energies, where the hydrodynamic picture fails! To be precise, the data collapse onto two different curves, one for mesons and another for baryons. As if 
this were not miraculous enough, we can make these two branches overlap perfectly by dividing both axes, the elliptic flow and the transverse kinetic energy, by the number of valence quarks in the hadron: now all hadrons fall onto a single universal scaling curve (right panel)! What this tells you is that the elliptic flow is carried by individual quarks, is the same for the light up and down and the heavier strange quarks, and is transferred to the hadrons at the point of hadronization by quark coalescence [13]. This is strong evidence for a key dynamical role being played by deconfined quarks and antiquarks in the QGP.

The observation of almost perfect fluid dynamical behaviour of the bulk of the matter at low $p_{T}$ requires strong coupling in the QGP. It is difficult and perhaps impossible to reproduce using QCD perturbation theory. But, for a special class of conformally symmetric quantum field theories (CFT), the strong-coupling limit can be analyzed with superstring theoretical methods, using the AdS/CFT duality [14] which says that solving the strongly coupled CFT is equivalent to solving classical gravitational equations of motion in 5-dimensional curved spacetimes with anti-de-Sitter metric! In such theories, Son and collaborators found that the shear viscosity to entropy ratio becomes particularly small and assumes a conjectured lower limit of $\frac{\eta}{s}=\frac{\hbar}{4 \pi k_{B}}$ [15]. A number of arguments, including some very recent viscous hydrodynamic calculations of the elliptic flow [10, 11, point strongly to an $\eta / s$ ratio for the QGP which is close to (i.e. not more than about a factor 5 larger than) this conjectured lower limit. This makes the QGP the most perfect (real, not quantum) liquid ever observed in the laboratory! The only other system that seems to be able to get close is that of trapped ultracold atoms in the unitary (i.e. strongly interacting) regime [16, 17].

As we moved from the BEVALAC via the SPS to RHIC, heavy-ion physics became mainstream. RHIC news now regularly captures the attention of the public and of scientists from other fields. Relativistic heavy-ion physics has always had a strong intellectual connection with astrophysics and cosmology (e.g. through quark stars, the conceptual similarities between the cosmological Big Bang and the Little Bangs created in heavy-ion collisions, and because RHIC explores the properties of the matter out of which our entire universe was initially made). As just noted, it now also has strong and fruitful bi-directional ties with the condensed matter physics of strongly coupled cold fermionic atoms, with high energy density physics through the physics of strongly coupled Coulomb plasmas, and with string theory and quantum gravity through the AdS/CFT correspondence which allows to explore strongly coupled gauge field theories with the help of their weakly coupled (classical) gravity duals. To foster such interdisciplinary ties has always been a hallmark of Maurice's work, at CERN and elsewhere. Naturally, he followed these developments with great enthusiasm until the end.

Epilogue. When I gave this talk I had just returned to CERN for a 1-year sabbatical. It was the first time for me at CERN without Maurice. I will always hold dear the spirited discussions I had with him during previous visits, and I will miss him this time around. But his legacy remains with us. It takes visionaries 
like Maurice Jacob to help create a young new field such as ultra-relativistic heavy-ion physics and nurture it to maturity. Those of us who have heard him tell stories about his children know how proud and supportive Maurice has always been of them. He took the same care of his brain-children.

Acknowledgements: I thank the organizers of the "Maurice Jacob Memorial Meeting", especially Luis Alvarez-Gaume, for giving me the opportunity to express my gratitude for what Maurice has done for my field of research, and Urs Wiedemann for helpful comments on the manuscript.

\section{References}

[1] T. D. Lee and G. C. Wick, Phys. Rev. D 9 (1974) 2291.

[2] J. C. Collins and M. J. Perry, Phys. Rev. Lett. 34, 1353 (1975).

[3] M. Jacob, "In search of quark matter: The new round of tests," Nucl. Phys. A 583, 13C (1995).

[4] U. Heinz and M. Jacob, arXiv:nucl-th/0002042.

[5] F. Karsch and E. Laermann, in Quark-Gluon Plasma 3, edited by R. C. Hwa and X. N. Wang (World Scientific, Singapore, 2004), p. 1 arXiv:hep-lat/0305025.

[6] The webcast archive of the events of that day and supporting material can be found at the URL http://newstate-matter.web.cern.ch/newstate-matter/.

[7] J. Adams et al. [STAR Collaboration], Phys. Rev. Lett. 92, 052302 (2004); ibid. 95, 122301 (2005).

[8] A. Adare et al. [PHENIX Collaboration], Phys. Rev. Lett. 98, 162301 (2007).

[9] B. Müller and J. L. Nagle, Ann. Rev. Nucl. Part. Sci. 56, 93 (2006).

[10] P. Romatschke and U. Romatschke, Phys. Rev. Lett. 99, 172301 (2007).

[11] H. Song and U. Heinz, Phys. Lett. B 658, 279 (2008).

[12] H. Appelshuser et al. [NA49 Collaboration], Phys. Rev. Lett. 80, 4136 (1998); A. M. Poskanzer and S. A. Voloshin, Nucl. Phys. A661, 341c (1999).

[13] V. Greco, C. M. Ko and P. Levai, Phys. Rev. Lett. 90, 202302 (2003); R. J. Fries, B. Müller, C. Nonaka and S. A. Bass, ibid. 90, 202303 (2003); D. Molnar and S. A. Voloshin, ibid. 91, 092301 (2003).

[14] J. M. Maldacena, Adv. Theor. Math. Phys. 2, 231 (1998) [Int. J. Theor. Phys. 38, 1113 (1999)]. 
[15] G. Policastro, D. T. Son and A. O. Starinets, Phys. Rev. Lett. 87, 081601 (2001); P. Kovtun, D. T. Son and A. O. Starinets, Phys. Rev. Lett. 94, 111601 (2005).

[16] J. Kinast, A. Turlapov, and J. E. Thomas, Phys. Rev. Lett. 94, 170404 (2005).

[17] T. Schäfer, Phys. Rev. A 76, 063618 (2007). 